\title{
Manuseio do Potencial Doador de Múltiplos Órgãos*
}

\author{
Care of the Potential Organ Donor
}

\author{
Tatiana H. Rech ${ }^{1}$, Édison Moraes Rodrigues Filho ${ }^{2}$
}

\section{RESUMO}

JUSTIFICATIVA E OBJETIVOS: O transplante de órgãos sólidos vem sendo considerado o tratamento de eleição para várias doenças terminais que afetam rim, pâncreas, fígado, coração e pulmão. Atualmente, o maior limitante às cirurgias dos transplantes é a escassez de órgãos. O objetivo deste estudo foi revisar aspectos fisiopatológicos da morte encefálica e resumir estratégias terapêuticas atuais, para o cuidado otimizado do doador, do que depende o sucesso dos transplantes.

CONTEÚDO: A morte encefálica é uma síndrome inflamatória que pode rapidamente produzir alterações deletérias nos órgãos dos potenciais doadores. Esse contexto de instabilidade hemodinâmica, metabólica e eletrolítica, exige do intensivista cuidados especiais com o doador de múltiplos órgãos.

CONCLUSÔES: O adequado conhecimento da complexa fisiopatologia envolvendo a morte encefálica é de fundamental importância para que se implemente de forma racional um protocolo de manuseio agressivo do potencial doador, o que certamente resultará num

1. Médica Rotineira da Unidade de Terapia Intensiva do Hospital Dom Vicente Scherer - Complexo Hospitalar Santa Casa de Porto Alegre, RS, Brasil. Mestre em Medicina Ciências Médicas pela Universidade Federal do Rio Grande do Sul.

2. Chefe da Unidade de Terapia Intensiva do Hospital Dom Vicente Scherer - Complexo Hospitalar Santa Casa de Porto Alegre, RS, Brasil. Mestre em Diagnóstico Genético e Molecular pela Universidade Luterana do Brasil.

${ }^{*}$ Recebido da Unidade de Terapia Intensiva do Hospital Dom Vicente Scherer, Porto Alegre, RS

Apresentado em 29 de janeiro de 2007

Aceito para publicação em 11 de abril de 2007

Endereço para correspondência:

Dra. Tatiana H. Rech

Rua General Couto de Magalhães, 1876/401

90540-130, Porto Alegre, RS

Fones: (51) 9959-5492 - 3337-6369

E-mail: tatianarech@terra.com.br

(C)Associação de Medicina Intensiva Brasileira, 2007 aumento de órgãos captados e do número de órgãos captados por doador, além da redução das taxas de disfunção primária dos enxertos transplantados.

Unitermos: Doador de órgãos, Morte encefálica, Tratamento hormonal, Transplante de órgãos.

\section{SUMMARY}

BACKGROUND AND OBJECTIVES: Organ transplantation has long been considered the treatment of choice for many end-stage organ diseases. As soon as transplantation turned to be a viable therapy, organ shortage became the major limitation for the procedures. Nowadays, there is an increasing imbalance between organ supply and demand. Apparently, the most promising way to increase organ supply is optimizing the care for the brain death organ donor. The objective of this manuscript was to review the pathophysiological aspects and therapeutic strategies for the optimized care of the potential organ donor.

CONTENTS: Brain death causes a massive catecholamine release, inducing a variety of deleterious effects that can threat organ perfusion. Studies have documented a sudden decrease in cortisol, insulin, thyroid and pituitary hormones. In this scenario of hemodynamic and metabolic instability, a special attention to the multiple organ donor support is required.

CONCLUSIONS: An extensive knowledge of the complex brain death pathophysiology is extremely important for the implementation of rational aggressive management protocols of the potential organ donor, aiming to increase the number of harvested organs and the number of organs harvested per donor.

Key Words: Brain death, Hormonal therapy, Organ donor, Organ transplantation.

\section{INTRODUÇÃO}

Tão logo os transplantes se firmaram como um tratamento viável, o seu maior limitante passou a ser a escassez de órgãos ${ }^{1}$, existindo hoje um desequilíbrio crescente entre a oferta e a demanda de órgãos ${ }^{2,3}$. 
Aparentemente, o caminho mais promissor para aumentar a oferta de órgãos é pela otimização do uso da sua principal fonte: o doador cadáver com morte encefálica, que pode oferecer simultaneamente oito órgãos para transplante, além de córneas, ossos e pele ${ }^{4}$. A morte encefálica produz liberação massiva de catecolaminas, causando uma série de efeitos deletérios que podem colocar em risco a perfusão de órgãos do potencial doador, além de provocar uma redução brusca nos níveis de cortisol, insulina, hormônios tireoidiano e antidiurético ${ }^{5,6}$.

\section{FISIOPATOLOGIA DA MORTE ENCEFÁLICA}

A morte encefálica é um processo complexo que altera de forma dramática a fisiologia e a bioquímica celulares de todos os sistemas orgânicos. A síndrome clínica da morte encefálica produz mudanças bruscas na pressão arterial, hipoxemia, hipotermia, coagulopatia, distúrbios eletrolítico e hormonal. É um estado inflamatório que leva a perturbações celulares e moleculares, capazes de afetar a função dos órgãos potencialmente disponíveis para transplante?

A morte encefálica representa o processo final de progressão da isquemia cerebral que evolui no sentido rostrocaudal até envolver regiões do mesencéfalo, ponte e medula, culminando com a herniação cerebral através do forâmen magno. O momento que precede a herniação cerebral é marcado por extremas elevações da pressão intracraniana, acompanhada da tríade de Cushing, que representa o esforço final do organismo na tentativa de manter a perfusão cerebral. A falência desse mecanismo promove a progressão da isquemia que, ao atingir a medula, interrompe a atividade vagal, levando a resposta autonômica simpática maciça, chamada de "tempestade autonômica"8. Essa estimulação simpática desenfreada tem curta duração e caracteriza-se por taquicardia, hipertensão, hipertermia e aumento acentuado do débito cardíaco. A gravidade dessas alterações está associada com a velocidade de instalação da hipertensão intracraniana e da herniação cerebral. Subseqüentemente, a tempestade autonômica cessa e o resultado é a perda do tônus simpático, com profunda vasodilatação e depressão da função cardíaca, que, se não tratadas, devem progredir para assistolia em torno de 72 horas ${ }^{9}$.

\section{Alterações Cardiovasculares}

A intensa liberação de catecolaminas durante a descarga autonômica produz grande vasoconstrição, que acarreta hipertensão arterial, taquicardia e aumento da demanda de oxigênio do miocárdio, podendo causar isquemia e necrose miocárdica, além de disritmias cardíacas. A morte encefálica associa-se às disfunções sistólica e diastólica do coração. A maciça liberação de catecolaminas na circulação periférica, após uma catástrofe neurológica, ocasiona uma resposta hiperdinâmica que mimetiza as crises hipertensivas do feocromocitoma ${ }^{9}$. Várias alterações eletrocardiográficas podem ser vistas: depressão ou elevação do segmento ST, ondas T invertidas, alargamento dos complexos QRS e prolongamento do intervalo $Q T^{10}$. Lesão subendocárdica é muito freqüente. Ao exame ecocardiográfico, até $43 \%$ dos corações de doadores com morte encefálica revelam algum grau de disfunção. Pode ocorrer diminuição da fração de ejeção e um mosaico de anormalidades de movimento da parede miocárdica. Alterações de contratilidade segmentar identificada por ecocardiografia não podem ser consideradas irreversíveis, não predizendo disfunção miocárdica em pacientes submetidos a transplante cardíaco ${ }^{11}$.

Na morte encefálica a disfunção cardíaca tem múltiplas causas. Logo após a descarga autonômica, segue-se um período de extrema vasodilatação, com hipotensão arterial grave. O colapso cardiovascular ocorre predominantemente em virtude dessa profunda vasodilatação e hipovolemia relativa ${ }^{12}$. Szabó ${ }^{13}$ sugeriu que as alterações cardíacas do doador são reflexos das condições da pré e pós-carga ventricular e da perfusão coronariana, não de lesão cardíaca irreversível em virtude da reação de Cushing inicial e depleção hormonal. A morte encefálica leva a profundas alterações de pós-carga em decorrência da vasodilatação extrema, que repercute na pré-carga, diminuída de formas relativa e absoluta; causando hipoperfusão coronariana. O colapso circulatório resultante desse mecanismo é, portanto, reversível, e a otimização da pré e pós-carga no doador resulta na melhora da contratilidade miocárdica, tornando viáveis para transplante, corações inicialmente considerados marginais.

A hipotensão arterial é a alteração fisiopatológica mais freqüente da morte encefálica. A vasodilatação produz grande aumento da capacitância do sistema vascular, produzindo hipovolemia relativa. A hipovolemia absoluta é conseqüência das perdas sangüíneas pelo trauma, da reanimação inadequada do doador, do uso de tratamento osmótico para hipertensão intracraniana, da diabete insipidus, além da diurese osmótica causada pela hiperglicemia e dos efeitos da hipotermia sobre a diurese ${ }^{14}$. 


\section{Alterações Pulmonares}

O pulmão é muito susceptível aos eventos que se seguem à morte encefálica. $\mathrm{O}$ aumento da permeabilidade vascular ocorre também no território pulmonar, como resposta às alterações inflamatórias que cursam com a morte encefálica. Pacientes em coma podem ter lesão pulmonar por trauma, pneumonite de aspiração e embolia gordurosa. Uma vez excluídas essas possibilidades, edema pulmonar neurogênico, embora infreqüente, deve ser considerado ${ }^{15}$.

A estimulação simpática maciça pode levar a graus extremos de venoconstrição pulmonar, favorecendo escape capilar de fluídos ricos em proteínas. A venoconstrição intensa interfere nas forças de Starling no pulmão e não é detectável por alterações na mensuração da pressão de oclusão da artéria pulmonar (POAP). Insuficiência ventricular esquerda aguda e reposição volêmica excessiva podem aumentar a pressão atrial esquerda e a pressão da artéria pulmonar, favorecendo a transudação ${ }^{16}$.

\section{Alterações Endócrinas}

A falência progressiva do eixo hipotalâmico-hipofisário evolui para um declínio gradual e inexorável das concentrações hormonais, principalmente do hormônio antidiurético (ADH). Diabetes insipidus ocorre em aproximadamente $80 \%$ desses pacientes e caracteriza-se por grandes volumes de diurese hiposmolar, com hipovolemia secundária, hipernatremia e hiperosmolaridade sérica. É importante causa de hipotensão e hipoperfusão tecidual, produzindo inúmeros distúrbios eletrolíticos além da hipernatremia, que contribuem para o desenvolvimento de disritmias cardíacas e depressão miocárdica. É mais freqüente nos pacientes vítimas de efeito de massa, sendo menos comum em vítimas de processos difusos como meningoencefalites e isquemia global, pois o lobo posterior da hipófise recebe sangue das artérias hipofisárias anteriores, que possuem trajeto extradural. O lobo anterior da hipófise, por sua compartimentalização dentro da sela túrcica, é normalmente poupado nas lesões cerebrais, o que explica a função hipofisária normal em alguns pacientes com morte encefálica ${ }^{17}$.

Alguns autores defendem que o verdadeiro hipotireoidismo está presente quando há diminuição de $T_{3}$ livre, que é o melhor indicador de disfunção do eixo tireoidiano-hipofisário. Diminuições bruscas nos níveis de triiodotironina $\left(\mathrm{T}_{3}\right)$ são constatadas logo após a morte encefálica e têm sido implicadas na redução da contratilidade cardíaca, com depleção de fosfatos de alta energia e mudança do metabolismo aeróbico para anaeróbico. Isso contribui para a piora da acidose metabólica e da perfusão tecidual dos órgãos do doador. A função tireoidiana pode revelar resultados laboratoriais característicos da síndrome do paciente eutireóideo. Como em muitas doenças graves, os níveis séricos de $\mathrm{T}_{3}-\mathrm{T}_{4}$ e $\mathrm{T}_{4}$ livre estão diminuídos ou no limite, mas os níveis de TSH estão normais ${ }^{18}$.

O fato de que níveis circulantes de prolactina, cortisol, hormônio do crescimento e hormônios gonadais não diminuem consideravelmente, confirma a impressão clínica que o pan-hipopituitarismo não está presente na fisiopatologia da morte encefálica. Porém, baixos níveis de cortisol podem ser observados, secundários à diminuição da liberação de ACTH pela hipófise anterior. Essa alteração, somada ao quadro de redução dos hormônios tireoidianos, contribui fortemente para a instabilidade hemodinâmica observada no doador ${ }^{19}$. Finalmente, a secreção de insulina também está comprometida, podendo produzir hiperglicemia. Aliado a isso, parece haver aumento da resistência periférica à insulina ${ }^{2,20}$.

\section{Alterações Hepáticas e da Coagulação}

No fígado ocorre depleção dos estoques de glicogênio e redução da perfusão sinusoidal hepática. Alterações das transaminases e das bilirrubinas são incomuns.

A coagulopatia é bastante freqüente. A lesão do tecido cerebral libera tromboplastina tecidual e outros substratos ricos em plasminogênio. Esses fatores, somados à hemorragia, transfusões, diluição dos fatores de coagulação, acidose e hipotermia, favorecem o desenvolvimento de coagulação intravascular disseminada ${ }^{21}$.

\section{Alterações da Temperatura}

A regulação hipotalâmica da temperatura é perdida com a morte encefálica. A vasodilatação extrema típica da síndrome, associada à inabilidade de tremer para produzir calor, além da infusão de grandes volumes de fluidos não aquecidos, resulta em diminuição da temperatura de forma muito rápida. A hipotermia induz a diversos efeitos deletérios, como disfunção cardíaca, disritmias, coagulopatia, desvio da curva de dissociação da hemoglobina para a esquerda e diurese induzida pelo frio ${ }^{22,23}$.

\section{Alterações Imunológicas}

A síndrome da morte encefálica é um estado inflamatório. A ativação de mediadores inflamatórios como complemento, tromboxanos e fatores leucocitários têm 
papel central nos eventos que permeiam a morte encefálica. À medida que o tempo passa, ocorre contínua infiltração inflamatória nos tecidos, o que aumenta a imunogenicidade dos enxertos. Detectam-se altos níveis de citocinas pró-inflamatórias em diversos órgãos, que desencadeiam e amplificam a resposta imunológica aguda no receptor. Comparações entre resultados de órgãos transplantados mostram que os episódios de rejeição aguda são mais comuns e mais graves em órgãos captados de doadores com morte encefálica do que naqueles de doadores vivos ${ }^{24}$. Na tabela 1 estão apresentadas as principais alterações fisiopatológicas da morte encefálica.

Tabela 1 - Incidência de Alterações Fisiopatológicas após a Morte Encefálica ${ }^{12}$

\begin{tabular}{ll}
\hline Hipotensão arterial & $81 \%$ \\
Diabetes insipidus & $78 \%$ \\
Coagulação intravascular disseminada & $28 \%$ \\
Disritmias cardíacas & $25 \%$ \\
Edema pulmonar & $18 \%$ \\
Acidose metabólica & $11 \%$ \\
\hline
\end{tabular}

\section{CUIDADOS INTENSIVOS COM O POTENCIAL DOADOR}

A morte encefálica causa múltiplos efeitos deletérios sobre o organismo, o que resulta em instabilidade cardiovascular, desarranjos metabólicos e hipoperfusão tecidual. Cuidados intensivos padronizados com o doador de múltiplos órgãos estão associados ao aumento do número de órgãos captados e à sua qualidade $^{25-27}$. O momento da declaração da morte encefálica é crítico, com mudanças drásticas das prioridades de tratamento. $\mathrm{O}$ tratamento voltado para a otimização da perfusão cerebral perde sentido, visto que os objetivos passam a ser a proteção e a perfusão dos órgãos especificamente. A sua prioridade é garantir o melhor suporte fisiológico possível para potencializar o sucesso dos órgãos transplantados. O manuseio agressivo do doador pode reverter a disfunção temporária de órgãos ${ }^{6}$.

\section{Cuidados Gerais}

Os cuidados gerais iniciam com a revisão das medicações prescritas. As utilizadas no tratamento do quadro neurológico não são mais necessárias. Devem-se suspender anticonvulsivantes, analgésicos, antitérmicos e diuréticos osmóticos. Antibióticos em uso devem sem mantidos ${ }^{28}$.

A assistência de enfermagem deve atender às necessidades fisiológicas básicas do potencial doador e, dentre todos os cuidados, os mais relevantes são manutenção de cabeceira elevada a $30^{\circ}$, mudanças de decúbito, aspiração de secreções pulmonares e cuidados com os cateteres. Pressão arterial, freqüência cardíaca, temperatura e oximetria de pulso devem ser mensuradas continuamente. As medidas da pressão venosa central (PVC), do débito, da densidade urinária e da glicemia capilar, devem ser realizadas a cada hora. O uso de cobertores e de fluidos aquecidos ajuda a prevenir a diminuição da temperatura ${ }^{22}$. Dietas por via enteral devem ser mantidas. Existem evidências de que oferecer nutrientes a órgãos específicos pode melhorar a função dos enxertos nos receptores. Isso tem maior relevância quando se trata de fígado e intestino ${ }^{7}$.

\section{Dosagens Laboratoriais}

A coleta de material para exames laboratoriais, incluindo hemograma, plaquetas, uréia, creatinina, sódio, potássio, cloro, magnésio, cálcio, fósforo, troponina, gasometria arterial e provas de coagulação, devem ser feitas regularmente de acordo com os protocolos estabelecidos. A tipagem sangüínea deve ser feita rapidamente, assim como o radiograma de tórax e o eletrocardiograma ${ }^{28}$.

\section{Cuidados Cardiovasculares}

A manutenção da estabilidade hemodinâmica é de fundamental importância para a viabilidade dos órgãos. Isso está bem evidente, tanto que se sabe que a captação do rim é maior quando coração e rins são captados juntos.

A hipertensão é incomum no doador, mas faz parte do quadro de herniação cerebral; por ser de curta duração, raramente precisa ser tratada. $O$ fármaco de escolha é o nitroprussiato de sódio.

O colapso circulatório, resultado da vasodilatação extrema, da hipovolemia, da disfunção ventricular e da depleção hormonal, deve ser tratado com vigor. A hipotensão arterial é mais freqüente na morte encefálica e na hipovolemia, sendo a primeira causa a ser avaliada. A correção da hipovolemia necessita de acesso venoso central para infusão de grandes volumes de líquidos e para as medidas da PVC. O objetivo é restaurar a euvolemia, pois hipervolemia é deletéria quando se considera a captação de pulmão. Volumes médios, em torno de quatros litros de cristalóides, podem ser necessários na reposição volêmica. Não há evidências convincentes de que cristalóides ou colóides sejam a melhor opção nessa situação. Soluções de Ringer com lactato são consideradas boas opções, em virtude de 
serem levemente hipotônicas. Quando a hipernatremia é muito grave, pode ser necessário utilizar solução fisiológica a $0,45 \%$. O uso de soluções de hidroxietilamido deve ser evitado, pois está associado à lesão tubular renal e à disfunção primária de enxertos renais. Colóides podem ser a melhor opção na reanimação de pacientes candidatos à doação de pulmão ${ }^{8,16}$.

Quando a hipotensão persiste a despeito da reposição volêmica adequada, é necessária a utilização de inotrópicos e vasopressores para restaurar a perfusão tecidual. O objetivo do uso de vasopressor é manter a pressão arterial média (PAM) em torno de $70 \mathrm{mmHg}$. O uso de vasopressores exige a monitoração invasiva da pressão arterial. O aumento progressivo do suporte deve ser acompanhado do escalonamento da monitoração.

A dopamina é recomendada pela maioria dos autores como primeiro fármaco a ser utilizado, porém não há estudos aleatórios definindo qual é o melhor vasopressor nessa situação. A dose de dopamina não deve ultrapassar a $10 \mu \mathrm{g} / \mathrm{kg} / \mathrm{min}$, principalmente quando se pretende captar coração. Noradrenalina e vasopressina são fármacos a serem consideradas quando persistir hipotensão ou evidências de hipoperfusão tecidual. $\mathrm{O}$ uso de altas doses de vasopressor tem sido associado à redução da sobrevida dos enxertos cardíacos e renais ${ }^{16,21}$. Os potenciais doadores devem ser submetidos a uma avaliação inicial com ecocardiograma. Os pacientes que já receberam volume adequadamente, nos quais os índices de perfusão permanecem inadequados e a fração de ejeção é menor que $45 \%$, devem ser avaliados quanto à colocação de cateter de artéria pulmonar para guiar o manuseio hemodinâmico ${ }^{29}$. Apesar do uso do cateter de Swan-Ganz ${ }^{30}$ ser cada vez mais questionado, a adequada interpretação das medidas obtidas, pode orientar a administração de volumes e fármacos vasoativos, especialmente em candidatos à doação de coração e pulmão. Após otimização hemodinâmica máxima, que inclui o uso de cateter de artéria pulmonar e reposição hormonal, um novo ecocardiograma pode mostrar meIhora da fração de ejeção, possibilitando o uso de corações inicialmente considerados marginais ${ }^{16}$.

Disritmias cardíacas são freqüentes nos doadores e podem comprometer o débito cardíaco. Bradicardias podem exigir o uso de marcapasso, pois não respondem ao uso de atropina. Taquicardia é habitual. O doador pode apresentar disritmias de todos os tipos, geralmente associadas a distúrbios eletrolíticos e ácido-básicos graves, e são tratadas de forma usual ${ }^{31}$. $\mathrm{Na}$ tabela 2 estão apresentados os principais objetivos hemodinâmicos no doador de múltiplos órgãos.
Tabela 2 - Objetivos Hemodinâmicos no Doador ${ }^{3}$

\begin{tabular}{lc}
\hline Freqüência cardíaca & $60-120 \mathrm{bpm}$ \\
Pressão arterial média & $70 \mathrm{mmHg}$ \\
Pressão arterial central & $8-12 \mathrm{mmHg}$ \\
Pressão de oclusão da artéria pul- & $6-10 \mathrm{mmHg}$ \\
monar & \\
Índice cardíaco & $>2,4 \mathrm{~L} / \mathrm{min} / \mathrm{m}^{2}$ \\
Resistência vascular sistêmica & $800-1200 \mathrm{dinas} / \mathrm{s} / \mathrm{cm}^{-5}$ \\
Índice do trabalho do ventrículo & $>15 \mathrm{~g} / \mathrm{kg} / \mathrm{min}^{-}$ \\
esquerdo & $>45 \%$ \\
Fração de ejeção &
\end{tabular}

\section{Reposição Hormonal}

Diabetes insipidus é, de longe, o distúrbio endócrino mais comum no paciente com morte encefálica. Ela é causada por grave redução na liberação de ADH pela hipófise, resultando em grande perda renal de água livre, produzindo hipernatremia. A hipovolemia e o estado hiperosmolar comprometem a hemodinâmica do doador e a função dos órgãos. Hipernatremia está relacionada à disfunção primária dos enxertos hepáticos ${ }^{7}$. A reposição volêmica de escolha nessa situação recai sobre soluções hipotônicas com o objetivo de manter o sódio abaixo de $150 \mathrm{mEq} / \mathrm{L}$. Freqüentemente, a diurese é tão abundante que a reposição volêmica não é suficiente para manter a euvolemia, necessitando-se associar o uso de reposição hormonal. A arginina-vasopressina tem sido considerada como agente de escolha para o tratamento da diabetes insipidus, porque, além do seu efeito antidiurético, tem potente efeito vasoconstritor. O uso de vasopressina está associado à melhora da função dos enxertos renal, pulmonar e cardíaco, razão pela qual alguns autores recomendam seu uso como fármaco vasopressor de primeira escolha no manuseio de potenciais doadores. A dose a ser utilizada deve ser titulada em função do débito urinário. Em alguns casos pode ser preciso associar o uso de 1-D-amino-8-arginina-vasopressina (DDAVP) à vasopressina, que tem efeito antidiurético muito mais potente, porém com efeito vasopressor negligenciável. A titulação da dose é orientada pelo volume de diurese, que deve ser mantido abaixo de $300 \mathrm{~mL} / \mathrm{h}$. O uso de DDAVP não está associado a nenhum efeito adverso na função primária de enxertos renais ${ }^{20,29}$.

Os níveis de vasopressina e cortisol podem se tornar indetectáveis após quatro horas da morte encefálica. Níveis de $T_{3}, T_{4}$ e insulina reduzem-se significativamente em algumas horas. Evidências recentes favorecem o uso do tratamento hormonal tríplice em pacientes com instabilidade hemodinâmica ${ }^{5}$. Estudo retrospectivo com mais de 10.000 pacientes demonstrou que a associação 
de metilprednisolona, vasopressina $\mathrm{e}_{3}$ ou $\mathrm{T}_{4}$ promovem estabilidade hemodinâmica nos doadores e aumenta substancialmente o número de órgãos transplantados por doador ${ }^{32}$. O uso do tratamento tríplice está relacionado a um aumento de $28 \%$ da probabilidade de um paciente tornar-se doador de coração e na melhora da função aguda dos enxertos cardíacos ${ }^{33}$.

A reposição de hormônios tireoidianos, apesar de permanecer indefinida, parece produzir efeitos benéficos no desempenho cardíaco dos doadores. Ocorre também melhora da perfusão tecidual, com mudança do metabolismo anaeróbico para aeróbico. A reposição de $T_{3}$ parece ter vantagens sobre a reposição de $T_{4}$, pois é mais potente e tem início de ação mais precoce. Defensores da utilização de hormônios tireoidianos justificam seu uso com base nos efeitos positivos sobre a entrada de cálcio transarcolêmica, sobre a miosina miocárdica e sobre a atividade da ATPase, efeitos que podem manter a contratilidade ventricular ${ }^{34}$. Estudo realizado por Novitzky e col. mostrou efeito dramático da infusão de $T_{3}$ em pacientes com morte encefálica hemodinamicamente instáveis ${ }^{35}$. Infusões de quatro horas de uma combinação de $T_{3}$, cortisol e insulina meIhoraram significativamente o estado circulatório, com melhora no débito cardíaco e redução na necessidade de agentes inotrópicos. Outros estudos falharam em documentar efeitos favoráveis da administração de $T_{3}$ sobre a contratilidade miocárdica.

O uso de metilprednisolona em altas doses no paciente com morte encefálica, melhora os parâmetros de oxigenação e aumenta a captação de pulmão ${ }^{36}$. A administração de corticóide é um preditor independente de sucesso na doação de pulmão, provavelmente por produzir redução nas citocinas inflamatórias, diminuindo a resposta aloimune no receptor. Altos níveis de citocinas inflamatórias são associados à disfunção primária dos enxertos. Grande estudo revelou redução de $10 \%$ na perda dos enxertos renais em um ano, em pacientes tratados com corticóide, quando comparados aos não tratados ${ }^{33}$. A discussão atual sobre os níveis adequados de cortisol em pacientes críticos sugere a administração de corticóide em paciente com choque não-responsivo, especialmente na vigência de eosinofilia relativa ou absoluta e na documentação de nível de cortisol randômico, inferior a 15 ou mesmo $25 \mu \mathrm{g} / \mathrm{dL}^{37}$. Hiperglicemia é muito comum em pacientes com morte encefálica e altas doses de insulina, por via venosa, podem ser necessárias para o controle glicêmico. $\mathrm{Na}$ tabela 3 estão apresentadas as doses recomendadas na reposição hormonal.
Tabela 3 - Protocolo de Reposição Hormonal ${ }^{20}$

\begin{tabular}{lc}
\hline Vasopressina & $1 \mathrm{U}$ em bolus, infusão $0,5-2,5 \mathrm{U} / \mathrm{h}$ \\
$\mathrm{T}_{3}$ & $4 \mathrm{\mu g}$ em bolus, infusão $3 \mu \mathrm{g} / \mathrm{h}$ \\
Metilprednisolona & $15 \mathrm{mg} / \mathrm{kg}$ em bolus \\
Insulina & Mínimo de $1 \mathrm{U} / \mathrm{h}$, titulada conforme a \\
& glicemia \\
\hline
\end{tabular}

\section{Estratégias Ventilatórias}

O transplante de pulmão é o mais limitado pela falta de órgãos. Apenas $20 \%$ dos doadores acabam se tornando doadores ${ }^{38}$. O pulmão do doador é particularmente vulnerável a lesões. A liberação de mediadores pró-inflamatórios que acompanha a morte encefálica tem papel central na disfunção pulmonar e pode ser agravada por estratégias ventilatórias deletérias e por reposição hídrica excessiva ${ }^{39}$.

O uso de protocolos para manuseio ventilatório resulta na captação de pulmões inicialmente considerados como inapropriados para doação. Cuidados ventilatórios de rotina são obrigatórios nesses pacientes, como aspiração de secreções pulmonares, fisioterapia respiratória e mudança de decúbito.

A reposição volêmica precisa ser criteriosa, sempre com o objetivo de manter a euvolemia. O aumento da permeabilidade capilar torna os pulmões muito vulneráveis a edema. Nesses casos, o alvo deve ser pressões de enchimento mais baixas.

Todos os doadores com morte encefálica necessitam de ventilação mecânica controlada. Podem-se utilizar regimes ventilatórios controlados a volume ou a pressão. Pressão controlada é usada para prevenir elevações nas pressões de pico, que resultam em barotrauma ${ }^{40}$. Estratégias ventilatórias protetoras reduzem a incidência de lesão pulmonar aguda e são indicadas também nos doadores. Volumes-correntes (VC) entre 6 e $8 \mathrm{~mL} / \mathrm{kg}$ são recomendados com o objetivo de manter a pressão de platô abaixo de $30 \mathrm{mmHg}$. A fração inspirada de $\mathrm{O}_{2}\left(\mathrm{FiO}_{2}\right)$ deve ser a menor possível para atingir uma pressão arterial de oxigênio $\left(\mathrm{PaO}_{2}\right)$ maior que $90 \mathrm{mmHg}$. A pressão positiva no final da expiração (PEEP) varia entre 5 e 10 $\mathrm{cmH}_{2} \mathrm{O}$, não devendo ultrapassar esses valores, pois pode ser causa de hipotensão. Gasometrias arteriais freqüentes são indispensáveis no manuseio ventilatório dos doadores, assim como radiogramas de tórax ${ }^{41}$.

\section{Tratamento Transfusional}

A anemia e a coagulopatia são bastante comuns nos doadores. Perdas sangüíneas persistentes podem piorar a coagulação. Não se sabe qual é o grau de anemia que se pode tolerar no doador; por isso, a maioria dos 
autores recomenda manter o hematócrito em torno de $30^{16,28,29}$, a despeito dos constantes questionamentos sobre a capacidade de transporte de oxigênio e de hemácias estocadas, do risco aumentado de imunossupressão e do risco de ofertar sangue $\mathrm{CMV}+$.

A coagulação intravascular disseminada resulta de múltiplos fatores, incluindo liberação de tromboplastina tecidual, hipotermia, coagulopatia dilucional e politransfusão. Deve-se suspender o uso de qualquer medicamento com efeito anticoagulante ou antitrombótico tão logo se identifique um potencial doador. Plasma fresco, plaquetas e crioprecipitado só estão indicados em caso de sangramento ativo.

\section{Manuseio Ácido-Básico e Eletrolítico}

Desordens ácido-básicas podem ser potencialmente graves nos doadores e comprometer a função de órgãos. Alcalose respiratória é geralmente conseqüência da hiperventilação e do tratamento diurético utilizado na tentativa de reduzir a pressão intracraniana e pode causar vasoconstrição coronariana e dissociação da curva de hemoglobina para a esquerda. Acidose metabólica é causada por hipoperfusão tecidual e leva à piora da contração cardíaca e a redução da resposta endotelial às catecolaminas, causando mais vasodilatação e hipotensão. O objetivo deve ser a obtenção do pH entre 7,35 e 7,45. O uso de bicarbonato de sódio pode ser deletério, agravando ainda mais a hipernatremia ${ }^{11}$.

Anormalidades nos níveis de sódio, potássio, magnésio, cálcio e fósforo são normalmente resultados de grandes perdas urinárias. Esses íons têm muitos papéis na fisiologia celular; portanto, as alterações das concentrações séricas devem ser vigorosamente tratadas. Dosagens eletrolíticas freqüentes são recomendadas ${ }^{42,43}$.

\section{CRITÉRIOS DE SELEÇÃO DO POTENCIAL DOADOR}

A crescente demanda por órgãos tem levado ao uso de doadores marginais. Contra-indicações absolutas à doação de órgãos incluem infecção pelo vírus HIV e HTLV; infecções virais sistêmicas, como sarampo, adenovírus e parvovírus; encefalite herpética; tuberculose pulmonar; doenças relacionadas a príons e neoplásicas ${ }^{44}$.

Doenças malignas impedem a doação de órgãos, com exceção de cânceres de pele não-melanoma e alguns tumores primários do sistema nervoso central (SNC). Metástases de tumores primários do SNC são raras, mas bem documentadas. Alguns tumores são de alto risco para metástases e a sua ocorrência deve excluir o uso de órgãos, como meduloblastoma, ependimoma, gliossarcoma, meningioma de alto grau, astrocitoma anaplásico e cordoma. Múltiplas craniotomias e a presença de derivações aumentam o risco de metástases extracranianas. É preciso, nesses casos, examinar cuidadosamente o local da craniotomia e a presença de linfonodos cervicais, além do trajeto da derivação e da presença de implantes peritoneais ${ }^{45}$.

Sorologias positivas para hepatite B e C são contraindicações relativas para o uso de órgãos de doadores cadavéricos. A presença de HBsAg + representa a presença de DNA viral no sangue e infecção. Nessa situação, a hepatite B será transmitida ao receptor; logo, esses órgãos não podem ser utilizados. Com o aumento da vacinação para hepatite $B$, há alta prevalência de anti-HBs+. Anti-HBs+ associado a HBsAg-e $\mathrm{HBcAg}$ - não contra-indica doação; HBcAg IgG+ pode doar se tiver HBsAg- e anti-HBs+. Hepatite $C$ não é mais uma conta-indicação à doação. A transmissão viral certamente ocorre com o transplante de órgãos sólidos, mas os resultados pós-transplante parecem ser aceitáveis. Não há aumento na mortalidade ou na morbidade a curto (um ano) e médio (cinco anos) prazos associado com o transplante de fígado de doadores HCV+ quando comparado a doadores HCV-, seja em receptores $\mathrm{HCV}+$, seja em HCV- ${ }^{46}$. A sobrevida dos enxertos renais em 10 anos também não é afetada pela sorologia de hepatite $\mathrm{C}$ do doador ${ }^{47}$. $\mathrm{O}$ receptor $\mathrm{HCV}+$ é o receptor ideal de órgãos $\mathrm{HCV}+$, mas as urgências na lista de espera precisam ser consideradas.

Citomegalovírus transmitido através de órgãos sólidos causa infecção e doença nos receptores, especialmente nos receptores CMV-. Profilaxia de rotina contra $\mathrm{CMV}$ reduz expressivamente a morbimortalidade associada à infecção por esse vírus ${ }^{47}$.

Sepse e choque séptico não inviabilizam a doação de órgãos, desde que o doador esteja recebendo antibiótico. Os transplantes de órgãos de doadores sépticos têm resultados tão bons quanto os de órgãos de doadores sem infecção, tanto em relação à morbidade quanto à mortalidade ${ }^{48}$. Num estudo com 268 doadores, não houve nenhum caso em que a bactéria isolada no sangue dos doadores fosse a mesma encontrada nas culturas dos receptores ${ }^{49}$. Fungemia também não é critério para exclusão de doadores.

A utilização de órgãos de doadores intoxicados é segura em casos de envenenamento por organofosforados, cianetos, ácido acetilsalisílico e metano ${ }^{50,51}$. 


\section{CONCLUSÃO}

Diante da crescente escassez de órgãos é essencial que otimize o uso de órgãos captados de doadores com morte encefálica, atualmente a principal fonte de órgãos para transplantes. A identificação do potencial doador, o entendimento da complexa fisiologia da morte encefálica e os cuidados intensivos para manter a perfusão adequada dos órgãos são responsabilidades do médico intensivista e fazem parte da boa prática da terapia intensiva.

\section{REFERÊNCIAS}

01. Beasley C - Maximizing donation. Transplantation Rev, 1999;13:31-39.

02. de Perrot M, Weder W, Patterson GA et al - Strategies to increase limited donor resources. Eur Respir J, 2004;23:477-482.

03. Joshi N - Aggressive organ donor management: more from less? Curr Opin Organ Transplantation, 2006;11:141-145.

04. Ramos $\mathrm{H}$ - Critical care management of the brain-dead organ donor. Curr Opin Organ Transplantation, 2002;7:70-75.

05. Novitzky D, Cooper DK, Rosendale JD et al - Hormonal therapy of the brain-dead organ donor: experimental and clinical studies. Transplantation, 2006;82:1396-1401.

06. Gasser M, Waaga AM, Laskowski IA et al - The influence of donor brain death on short and long-term outcome of solid organ allografts. Ann Transplant, 2000;5:61-67.

07. Tuttle-Newhall JE, Collins BH, Kuo PC et al - Organ donation and treatment of the multi-organ donor. Curr Probl Surg, 2003;40:266-310.

08. Hevesi ZG, Lopukhin SY, Angelini G et al - Supportive care after brain death for the donor candidate. Int Anesthesiol Clin, 2006;44:21-34.

09. Jiang JP, Downing SE - Catecholamine cardiomyopathy: review and analysis of pathogenetic mechanisms. Yale J Biol Med, 1990;63:581-591.

10. Mayer SA, LiMandri G, Sherman D et al - Electrocardiographic markers of abnormal left ventricular wall motion in acute subarachnoid hemorrhage. J Neurosurg, 1995;83:889-896.

11. Dujardin KS, McCully RB, Wijdicks EF et al - Myocardial dysfunction associated with brain death: clinical, echocardiographic, and pathologic features. J Heart Lung Transplant, 2001;20:350-357.

12. Smith $M$ - Physiologic changes during brain stem death--lessons for management of the organ donor. J Heart Lung Transplant, 2004;23:(Supp19):S217-S222.

13. Szabo G - Physiologic changes after brain death. J Heart Lung Transplant 2004;23:(Suppl9)S223-S226.

14. Powner DJ, Darby JM - Management of variations in blood pressure during care of organ donors. Prog Transplant, 2000;10:25-30.

15. Simon RP - Neurogenic pulmonary edema. Neurol Clin, 1993;11:309323.

16. Wood KE, Becker BN, McCartney JG et al - Care of the potential organ donor. N Engl J Med, 2004; 351:2730-2739.

17. Howlett TA, Keogh AM, Perry L et al - Anterior and posterior pituitary function in brain-stem-dead donors. A possible role for hormonal replacement therapy. Transplantation, 1989;47:828-834.

18. Cooper DK, Basker M - Physiologic changes following brain death Transplant Proc, 1999;31:1001-1002.

19. Dimopoulou I, Tsagarakis S, Anthi A et al - High prevalence of decreased cortisol reserve in brain-dead potential organ donors. Crit Care Med, 2003;31:1113-1117.

20. Phongsamran PV - Critical care pharmacy in donor management. Prog Transplant, 2004;14:105-111.

21. Edgar PB, Bonner S - Management of the potential heart-beating organ donor. Continuing Education in Anaesthesia Crit Care, 2004;4:86-90.

22. Powner DJ, Reich HS - Regulation of coagulation abnormalities and temperature in organ donors. Prog Transplant, 2000;10:146-151.

23. Dosemeci L, Yilmaz M, Cengiz M et al - Brain death and donor management in the intensive care unit: experiences over the last 3 years. Transplant Proc, 2004;36:20-21.
24. Nijboer WS, van der Hoeven JAB, Ploeg RJ - Effect of brain death and donor treatment of organ inflammatory response and donor organ viability. Curr Opin Organ Transplant, 2004;9:110-115.

25. Rosendale JD, Chabalewski FL, McBride MA et al - Increased transplanted organs from the use of a standardized donor management protocol. Am J Transplant, 2002;2:761-768.

26. Helms AK, Torbey MT, Hacein-Bey L et al - Standardized protocols increase organ and tissue donation rates in the neurocritical care unit. Neurology, 2004;63:1955-1957.

27. Santiago C, Gomez P, Olivares J et al - Evaluation of organ procurement in an area under the influence of a training program. Transplant Proc, 2005;37:3649-3650.

28. Powner DJ, Darby JM, Kellum JA - Proposed treatment guidelines for donor care. Prog Transplant, 2004;14:16-26.

29. Shemie SD, Ross H, Pagliarello $\mathrm{J}$ et al - Organ donor management in Canada: recommendations of the forum on Medical Management to Optimize Donor Organ Potential. CMAJ, 2006;174:S13-S32.

30. Wheeler AP, Bernard GR, Thompson BT et al - Pulmonary-artery catheter versus central venus catheter to guide treatment of acute lung injury. N Engl J Med, 2006; 354: 2213-2224.

31. Powner DJ, Crommett JW - Advanced assessment of hemodynamic parameters during donor care. Prog Transplant, 2003;13:249-257.

32. Rosendale JD, Kauffman HM, McBride MA et al - Aggressive pharmacologic donor management results in more transplanted organs. Transplantation, 2003;75:482-487.

33. Rosendale JD, Kauffman HM, McBride MA et al - Hormonal resuscitation yields more transplanted hearts, with improved early function. Transplantation, 2003;75:1336-1341.

34. Novitzky D, Human PA, Cooper DK - Inotropic effect of triiodothyronine following myocardial ischemia and cardiopulmonary bypass: an experimental study in pigs. Ann Thorac Surg, 1988;45:50-55.

35. Novitzky D, Cooper DK, Morrell D et al - Change from aerobic to anaerobic metabolism after brain death, and reversal following triiodothyronine therapy. Transplantation, 1988;45:32-36.

36. Follette DM, Rudich SM, Babcock WD - Improved oxygenation and increased lung donor recovery with high-dose steroid administration after brain death. J Heart Lung Transplant, 1998;17:423-429.

37. Cooper MS, Stewart PM - Corticosteroid insufficiency in acutely ill patients. N Engl J Med, 2003;348:727-734.

38. McGiffin DC, Zorn GL Jr, Young KR Jr et al - The intensive care unit oxygen challenge should not be used for donor lung function decisionmaking. J Heart Lung Transplant, 2005;24:1902-1905.

39. Mascia L, Bosma K, Pasero D et al - Ventilatory and hemodynamic management of potential organ donors: an observational survey. Crit Care Med, 2006;34:321-327.

40. Powner DJ, Delgado E - Using pressure-limited mechanical ventilation in caring for organ donors. Prog Transplant, 2001;11:174-179.

41. Powner DJ, Darby JM, Stuart SA - Recommendations for mechanical ventilation during donor care. Prog Transplant, 2000;10:33-38.

42. Powner DJ, Kellum JA - Maintaining acid-base balance in organ donors. Prog Transplant, 2000;10:98-103.

43. Powner DJ, Kellum JA, Darby JM - Abnormalities in fluids, electrolytes, and metabolism of organ donors. Prog Transplant, 2000;10:88-94.

44. Rosengard BR, Feng S, Alfrey EJ, et al. Report of the Crystal City meeting to maximize the use of organs recovered from the cadaver donor. Am J Transplant, 2002;2:701-711.

45. Cavaliere RS, Donor D - Transmission of Primary Brain Tumors: A Neurooncologic Perspective. Transplantation Rev, 2004;18:204-213.

46. Williams TJ, Snell GI - Organ procurement--strategies to optimize donor availability. Semin Respir Crit Care Med, 2001;22:541-550.

47. Garrity ER Jr, Boettcher H, Gabbay E - Donor infection: an opinion on lung donor utilization. J Heart Lung Transplant, 2005;24:791-797

48. Zibari GB, Lipka J, Zizzi $\mathrm{H}$ et al - The use of contaminated donor organs in transplantation. Clin Transplant, 2000;14:397-400.

49. Cohen J, Michowiz R, Ashkenazi T et al - Successful organ transplantation from donors with Acinetobacter baumannii septic shock. Transplantation, 2006;81:853-855.

50. Lopez-Navidad A, Caballero F, Gonzalez-Segura C et al - Short- and long-term success of organs transplanted from acute methanol poisoned donors. Clin Transplant, 2002;16:151-162.

51. Duque $\mathrm{E}$, Duque J, Henao $\mathrm{J}$ et al - Organs transplanted from intoxicated donors. Transplant Proc, 2004;36:1632-1633. 